\title{
Seeing is believing
}

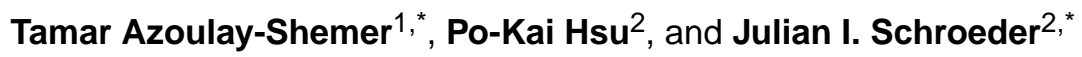

${ }^{1}$ Department of Plant Sciences and Genetics in Agriculture, R. H. Smith Faculty of Agriculture, The Hebrew University of Jerusalem, Rehovot, Israel

2Division of Biological Sciences, University of California San Diego, La Jolla, CA 92093-0116, USA

\begin{abstract}
Gibberellins (GAs) control key growth and developmental processes in plants. Real-time monitoring of GA concentrations in living tissues is critical for understanding the actions of this hormone class. A first-generation optogenetic GA-nano-indicator now illuminates the effects of GA levels on cell length and light signalling.
\end{abstract}

Gibberellic acids, also known as gibberellins (GAs), are a class of mobile hormones central for plant development and environmental responses. They have important functions in a broad array of physiological processes, including seed germination, stem and root elongation, flowering and senescence ${ }^{1}$. Moreover, increased yields in staple crops during the previous century's 'Green Revolution' are directly linked to GA concentrations in plant tissues ${ }^{2}$. Endogenous and exogenous factors have been predicted to affect intracellular GA levels in the cytoplasm and nucleus, where the GA signal is perceived. Studies have suggested that GA concentrations are dynamic in plant tissues, but direct real-time GA reporters have not been available to test cellular spatiotemporal synthesis and distribution models until now.

The current methods to investigate the regulation of GA levels in plants include expression profiles of GA-regulated genes, monitoring GA-induced proteolysis of the GFP-RGA reporter ${ }^{3}$, and GA quantification by gas chromatography/mass spectrometry (GC/MS). But these approaches do not easily allow cellular and sub-cellular resolution or direct real-time temporal analyses, which are essential for understanding how GA distribution at the tissue scale is controlled and how these patterns, in turn, can regulate plant growth and development. In this issue of Nature Plants, Rizza et al. report the development of a firstgeneration real-time GA-FRET (Förster/fluorescence resonance energy transfer) nanoreporter as a tool to investigate the in vivo distribution of $\mathrm{GAs}^{4}$.

Real-time quantitative detection of metabolites and hormones within cells represents a challenge in all fields of biology. Ratiometric small-molecule-binding nano-reporters, in

\footnotetext{
*Azoulay-Shemer@mail.huji.ac.il; jischroeder@ucsd.edu.

Competing interests

The authors declare no competing financial interests.
} 
particular those engineered as FRET reporters, function by means of conformationalchange-dependent energy transfer between two light-emitting molecules. Several FRET biosensors have been designed and used in living plants to visualize changes in intracellular calcium, sugars, abscisic acid and nitrate transport ${ }^{5-7}$. Now it is the turn of GA dynamics to be revealed, as Rizza et al. document, in real-time, the spatiotemporal distribution of the hormone in vivo. These analyses show correlation of elevated GA concentrations in the nucleus, and stimulation of cell elongation in roots and in dark-grown hypocotyls (Fig. 1). The authors further verify a model showing feedback regulation of GA levels by phytochrome-dependent light signalling through the phytochrome interacting factors (PIFs).

To generate a gibberellin biosensor, Rizza and colleagues screened for potential biosensor constructs based on a fusion protein of GA receptor GID1 (Gibberellin Insensitive Dwarf1) ${ }^{8}$ and the N-terminal domain of DELLA proteins that interact with the GID1 receptor upon GA binding ${ }^{9}$, fused to fluorescent proteins capable of FRET. After a comprehensive screening for potential GA-responsive reporters in yeast extracts, from many combinations of linkers and FRET pairs, one reporter was selected, further optimized and named Gibberellin Perception Sensor 1 (GPS1). Characterization of the GPS1 reporter showed defined FRET ratio changes in response to the bioactive GA isomers GA1, GA3 and GA4, and a preferential affinity for GA4, the main bioactive GA.

Rizza et al. generated stable transgenic Arabidopsis lines expressing a nuclear-targeted variant of GPS1 (nlsGPS1), enabling a pleasing visualization of GA patterns in individual cells and tissues, including roots, stamen filaments and hypocotyls (Fig. 1). GPS1 also showed some limitations. First, seedling growth analysis of these transgenic plants showed a reduced sensitivity to the GA biosynthesis inhibitor paclobutrazol, suggesting that the GAFRET biosensor has some activity in vivo. Second, although GPS1 showed interaction with high affinity to GA4, GPS1 also interacts with a lower affinity with other less active GAs. Finally, GA binding to GPS1 is either irreversible or has very slow dissociation rates for GAs, suggesting that GPS1 cannot be used to measure rapid GA degradation or removal from cells. Despite these hindrances, which are expected in the first version of a bioreporter, this study demonstrates that increases in GA levels can be measured at cellular resolution in living plant tissues and that biological questions can be addressed.

GAs are involved in the regulation of root cell length. Previous research has suggested accumulation of exogenous GA in endodermal cells of the root elongation zone as a key site of GA action in root elongation ${ }^{10}$. Using GPS1, Rizza et al. show beautifully visualized GA gradients along the growth axis of Arabidopsis hypocotyls and roots (Fig. 1). Furthermore, GA-dependent FRET is detected in root epidermal and cortical cells in addition to the endodermis. Additionally, exogenously applied GA4, but not GA1 or GA3, was found to accumulate preferentially in the elongation zone. These results lead to the hypothesis that the mechanisms governing accumulation of exogenously applied GAs can effectively discriminate between GAs, and preferentially accumulate GA4, the dominant bioactive GA in Arabidopsis, over GA3 or GA1.

Light is known to regulate GA levels. Light induces germination by causing GA accumulation in Arabidopsis seeds. Activation of phytochrome photoreceptors leads to the 
degradation of PIFs that are repressors of GA biosynthetic genes and inducers of GA degradation genes. In contrast, however, in hypocotyls, light decreases GA levels, whereas in the dark GA levels increase and induce cell elongation (Fig. 1). Using GPS1, Rizza et al. examine GA-dependent cell elongation mechanisms in roots, hypocotyls and stamen filaments. A mechanism is revealed in hypocotyls in which the intracellular GA concentration is upregulated by PIFs in the dark and phytochrome degradation of PIFs lowers GA in the light. These analyses demonstrate the power of direct cellular visualization of GAs.

Plant hormones were discovered through their central roles in plant growth and development. Many studies have hypothesized possible time-dependent changes and gradients in hormone concentrations in cells and across tissues. But direct real-time measurements of hormone levels in tissues are needed and will undoubtedly result in surprises and new findings. In addition to developing a first-generation GA nano-reporter, Rizza et al. have shown the applicability of the GPS1-FRET reporter in several tissues, under changing environmental conditions and in mutant backgrounds. Thus, GPS1 can be used to address biological questions and offer new insights. In the future, further engineering of GPS nano-reporters with accelerated dissociation rates, and altered selectivities and affinities for GAs, could also help to address open questions on localized conditional GA synthesis, transport and signalling.

\section{References}

1. Hedden, P., Thomas, SG. Annual Plant Reviews, Volume 49: The Gibberellins. Wiley; Chichester: 2016.

2. Hedden P. Trends Genet. 2003; 19:5-9. [PubMed: 12493241]

3. Silverstone AL, et al. Plant Cell. 2001; 13:1555-1566. [PubMed: 11449051]

4. Rizza, A., Walia, A., Lanquar, A., Frommer, WB., Jones, AM. Nat Plants. 2017. http://dx.doi.org/ 10.1038/s41477-017-0021-9

5. Okumoto S, Jones A, Frommer WB. Annu Rev Plant Biol. 2012; 63:663-706. [PubMed: 22404462]

6. Waadt R, et al. Bioessays. 2015; 37:1338-1349. [PubMed: 26577078]

7. Ho CH, Frommer WB. eLife. 2014; 3:e01917. [PubMed: 24623305]

8. Ueguchi-Tanaka M, et al. Nature. 2005; 437:693-698. [PubMed: 16193045]

9. Ueguchi-Tanaka M, et al. Plant Cell. 2007; 19:2140-2155. [PubMed: 17644730]

10. Ubeda-Tomas S, et al. Nat Cell Biol. 2008; 10:625-628. [PubMed: 18425113] 




Fig. 1. Real-time monitoring of GA concentrations in individual cells in plant tissues The nano-reporter GPS1 targeted to nuclei (nlsGPS1) is used in plant hypocotyls (top and middle) and in roots (bottom) to monitor GA concentrations with cellular resolution. The pseudo-coloured calibration bar (nlsGPS1 emission ratio) indicates nuclear GA levels. Note that the dark-grown seedling (left, top) shows higher GA concentrations in hypocotyl cells than the light-grown seedling (middle), consistent with GA stimulation of hypocotyl growth. GPS1-FRET ratios in root tips indicate a GA gradient (bottom) that positively correlates with root cell length. Fluorescence images reproduced from ref. ${ }^{4}$. 